This item was submitted to Loughborough's Research Repository by the author.

Items in Figshare are protected by copyright, with all rights reserved, unless otherwise indicated.

\title{
Hydro-acoustic precursors of gravity waves generated by surface pressure disturbances localised in space and time
}

PLEASE CITE THE PUBLISHED VERSION

http://dx.doi.org/10.1017/jfm.2014.398

\section{PUBLISHER}

(c) Cambridge University Press

\section{VERSION}

AM (Accepted Manuscript)

\section{PUBLISHER STATEMENT}

This work is made available according to the conditions of the Creative Commons Attribution-NonCommercialNoDerivatives 4.0 International (CC BY-NC-ND 4.0) licence. Full details of this licence are available at: https://creativecommons.org/licenses/by-nc-nd/4.0/

\section{LICENCE}

CC BY-NC-ND 4.0

\section{REPOSITORY RECORD}

Renzi, Emiliano, and F. Dias. 2019. "Hydro-acoustic Precursors of Gravity Waves Generated by Surface Pressure Disturbances Localised in Space and Time”. figshare. https://hdl.handle.net/2134/17135. 


\title{
Hydro-acoustic precursors of gravity waves generated by surface pressure disturbances localised in space and time
}

\author{
Emiliano Renzi ${ }^{1} \dagger$, and F. Dias ${ }^{1,2}$ \\ ${ }^{1}$ UCD School of Mathematical Sciences, University College Dublin, Belfield, Dublin 4, Ireland \\ ${ }^{2}$ CMLA, Ecole Normale Supérieure de Cachan, 94235 Cachan, France
}

(Received ?; revised ?; accepted ?. - To be entered by editorial office)

We consider the mechanics of coupled underwater-acoustic and surface-gravity waves generated by surface pressure disturbances in a slightly compressible fluid. We show that pressure changes on the ocean surface, localised in space and time, can induce appreciable underwater compression waves which are precursors of the surface gravity waves. Although the physical properties of acoustic-gravity waves have already been discussed in the literature, such dynamics was not investigated in previous studies. We derive new results for the underwater compression wave field and discuss the dynamics of its generation and propagation. This work could lead to the design of innovative alert systems for coastal flooding management.

Key words:

\section{Introduction}

In this paper, we show that the generation of transient surface gravity waves by sudden, localised surface pressure perturbations is associated with the existence of underwater acoustic precursors directly coupled with the gravity waves. This result advances previous incompressible and compressible models of ocean surface waves.

The vast majority of wave theories usually neglect the effect of compressibility on the generation and propagation of ocean surface waves. This is a safe assumption for many applications in ocean engineering (Mei et al. 2005). However, a quick analysis with the linearised theory of compressible water waves shows that propagating acoustic-gravity modes exist in addition to the surface gravity wave when $f>f_{0}=c /(4 h)$, where $f$ is the acoustic-gravity wave frequency, $c$ is the speed of sound in water and $h$ the bottom depth (Stiassnie 2010). Acoustic-gravity waves of frequency $f>f_{0}$ propagate at a speed of the order of the speed of sound in water and are much faster than the leading surface gravity waves (Lighthill 1978; Mei et al. 2005). This appealing feature of acoustic-gravity waves has only recently been considered in ocean engineering applications, like for example tsunami early warning (Stiassnie 2010; Sammarco et al. 2013). Indeed, recent analysis of hydrophone data has shown the occurrence of underwater acoustic noise directly coupled with violent atmospheric perturbations (Wilson \& Makris 2008). This suggests that compressibility needs to be included into the theory of transient wave generation by surface pressure variations.

$\dagger$ Email address for correspondence: emiliano.renzi@ucd.ie 
The physical properties of acoustic-gravity waves in compressible water have been already discussed in the past (Stoneley 1926; Longuet-Higgins 1950; Hasselmann 1963; Guo 1987; Ardhuin \& Herbers 2013; Ardhuin et al. 2013). However, except for Hasselmann (1963)'s statistical approach and Guo (1987)'s deep-water attempt, little attention has been paid to the hypothesis that underwater compression waves can be generated together with gravity waves directly by sudden pressure variations and gustiness present in turbulent air masses during storms or cyclones. Previous Authors considered surface pressure patterns caused by second-order interaction of gravity waves, neglecting the first-order effects of compressibility (Ardhuin \& Herbers 2013). They showed that nearsurface nonlinear hydrodynamic interactions of gravity waves generate pseudo-Rayleigh waves travelling at large depths and acoustic-gravity modes propagating closer to the ocean surface (Ardhuin et al. 2013). The direct transient response of a compressible fluid to a surface pressure field localised in space and time did not receive much attention, despite its potential impact in practical applications. Such a dynamics strongly depends on the previously neglected first-order compressibility and hence is related to a different phenomenon. We shall refer to the first-order acoustic-gravity modes as "hydro-acoustic" (HA) waves (Stiassnie 2010; Sammarco et al. 2013) to distinguish them from the secondorder pseudo-Rayleigh (R) and acoustic-gravity (AG) modes (Ardhuin \& Herbers 2013; Ardhuin et al. 2013). On the one hand, R and AG waves are the effect of interacting surface gravity waves. On the other hand, HA waves are directly interconnected to the gravity waves as both are induced by the same source, be it a ground motion (as in the case of tsunamis, see Stiassnie 2010) or a surface pressure variation. In other words, HA waves are generated together with (and are not a consequence of) surface gravity waves. We shall show that, due to the peculiar co-generation mechanism and different speeds of propagation in water, HA waves can be interpreted as the forerunners of surface gravity waves generated by surface pressure disturbances localised in space and time.

This paper aims: (i) To formalise the problem with a compressible Cauchy-Poisson system and to show that the latter does admit HA waves $(\S 2)$; (ii) To derive new formulae for the HA pressure field $(\S 3)$; (iii) To show that HA waves can be regarded as the Sommerfeld precursors (or forerunners) of the gravity surface waves generated by sudden surface pressure disturbances $(\S 4)$. The practical aspects of this fact are significant, in that HA waves could be used for the early detection of rogue waves or surges generated by atmospheric perturbations approaching coastal areas.

\section{Mathematical model}

\subsection{Governing equations}

Consider an ocean of constant depth $h$ which extends infinitely along the horizontal direction $x$. Assume that the characteristic extension of the surface pressure disturbance along the longitudinal axis $x$ is much smaller than that along the transverse direction $y$, like e.g. in a trailing squall line of a cyclone. Therefore the problem can be investigated in the two-dimensional vertical plane $(x, z)$. The $z$ axis rises from the unperturbed water level $z=0$ and is positive upwards, the bottom being at $z=-h$. Assume that the fluid is inviscid and weakly compressible and that the water motion is irrotational. The water density $\rho=\rho_{0}+\rho^{\prime}$ can be regarded as the sum of a constant ambient density $\rho_{0}$ and a perturbation $\rho^{\prime} \ll \rho_{0}$. Assume that the fluid is barotropic, so that the pressure $p=p(\rho)$. Taylor-expanding the latter expression about the constant density $\rho_{0}$ yields

$$
p(\rho)=p\left(\rho_{0}\right)+c^{2} \rho^{\prime}+O\left(\rho^{\prime 2}\right),
$$


where

$$
\mathrm{d} p / \mathrm{d} \rho=c^{2}
$$

is the square of the (assumed constant) speed of sound in water (Lighthill 1978). In the following, all physical quantities of order $O\left(\rho^{\prime 2}\right)$ and higher will be neglected upon the hypothesis of weakly compressible fluid. Irrotationality of the wave field allows the existence of a velocity potential $\phi(x, z, t)$ such that the velocity vector $\mathbf{v}$ satisfies $\mathbf{v}(x, z, t)=\nabla \phi$, $t$ being time and $\nabla f(x, z, t)=\left(f_{x}, f_{z}\right)$. Subscripts denote differentiation with respect to the relevant variable. The equation of mass conservation yields

$$
\rho_{t}^{\prime}+\rho_{0} \nabla^{2} \phi=0 .
$$

In the latter expression, the convective term $\mathbf{v} \cdot \nabla \rho^{\prime}$ and the quantity $\rho^{\prime} \nabla \cdot \mathbf{v}$ have been neglected as higher-order effects of compressibility (Lighthill 1978). The linearised momentum equation in Bernoulli's form reads

$$
g z+\int \frac{d p}{\rho}+\phi_{t}=C(t)
$$

where $g$ is the acceleration due to gravity. $C(t)=0$ since no standing waves are expected to be generated (Ardhuin \& Herbers 2013). Combination of (2.4) with (2.1)-(2.3) yields the well-known wave equation

$$
\phi_{t t}=c^{2} \nabla^{2} \phi, \quad x \in \mathbb{R}, z \in(-h, 0) .
$$

On the free surface, the linearised kinematic boundary condition reads

$$
\zeta_{t}=\phi_{z}, \quad z=0,
$$

$\zeta(x, t)$ being the free-surface elevation. Applying the Bernoulli equation (2.4) on the linearised free surface yields the dynamic boundary condition

$$
\phi_{t}+g \zeta=-P_{a}(x, t) / \rho_{0}, \quad z=0,
$$

where $P_{a}$ is the prescribed localised and transient pressure disturbance acting on the free surface. We require that $P_{a} \rightarrow 0$ as $|x| \rightarrow \infty$ and as $t \rightarrow \infty$. Physically, $P_{a}$ offers a simple representation of the action of convective downdrafts generated by rain-cooled air, fast sinking inside the squall line. Finally, at the bottom the no-flux condition reads

$$
\phi_{z}=0, \quad z=-h \text {. }
$$

We assume that the external forcing initiates at $t=0$ and that the fluid is unperturbed for all $t<0$. Then the following initial conditions apply:

$$
\phi(x, z, 0)=0, z \in[-h, 0] ; \quad \zeta(x, 0)=0 .
$$

As a consequence, (2.7) yields

$$
\phi_{t}(x, 0,0)=-P_{a}(x, 0) / \rho_{0} .
$$

Unlike for an incompressible fluid, when the fluid is compressible a sudden pressure change on the free surface is not transmitted instantaneously underneath. Therefore we need to prescribe the state of all fluid particles in the fluid domain at $t=0$. Below the free surface, the fluid is still at rest at $t=0$, so that the pressure there is distributed hydrostatically, i.e.

$$
\phi_{t}(x, z, 0)=0, \quad z \in[-h, 0) .
$$

This condition is peculiar to the compressible problem and does not appear in the incompressible case (see e.g. Mei et al. 2005). 


\subsection{Solution}

The set of equations (2.5)-(2.11) represents a Cauchy-Poisson problem of acoustic-gravity wave generation by a surface pressure disturbance in a compressible fluid. Let us now introduce the Laplace transform of the velocity potential

$$
\bar{\phi}(x, z ; s)=\int_{0}^{\infty} \phi(x, z, t) e^{-s t} \mathrm{~d} t ; \quad \phi(x, z, t)=\frac{1}{2 \pi i} \int_{\Gamma} \bar{\phi}(x, z ; s) e^{s t} \mathrm{~d} s,
$$

where $\Gamma$ is a vertical line lying on the right of the singularities of $\bar{\phi}(x, z ; s)$ in the complex $s$ plane (Mei 1997). Note that no Sommerfeld radiation condition can be required on $\phi$, since the transient nature of the perturbation prevents the system from reaching a steady sinusoidal state of outgoing radiation at any finite time (Mei et al. 2005). Instead, because of the spatio-temporal localisation of the phenomenon under analysis, we expect that the disturbance generated at $t=0$ by the pressure acting on the free surface vanishes far from the source at all times. This in turn yields $\phi(x, y, t) \rightarrow 0$ as $|x| \rightarrow \infty$. As a consequence, the Fourier transform in the unbounded $x$ domain can be used with no mathematical ambiguity:

$$
\hat{\bar{\phi}}(z ; k, s)=\int_{-\infty}^{\infty} \bar{\phi}(x, z ; s) e^{-\mathrm{i} k x} \mathrm{~d} x ; \quad \bar{\phi}(x, z ; s)=\frac{1}{2 \pi} \int_{-\infty}^{+\infty} \hat{\bar{\phi}}(z ; k, s) e^{\mathrm{i} k x} \mathrm{~d} k .
$$

Transforming the system of equations (2.5)-(2.8) with (2.12)-(2.13) and applying the initial conditions $(2.9)-(2.11)$ yields the following boundary-value problem for the double Fourier-Laplace transform of the velocity potential:

$$
\begin{aligned}
\hat{\bar{\phi}}_{z z}-\left(k^{2}+\frac{s^{2}}{c^{2}}\right) \hat{\bar{\phi}} & =0, \quad z \in(-h, 0) \\
\hat{\bar{\phi}}_{z}+\frac{s^{2}}{g} \hat{\bar{\phi}}=-\frac{s}{\rho_{0} g} \hat{\bar{P}}_{a}(k, s), \quad z & =0 \\
\hat{\bar{\phi}}_{z} & =0, \quad z=-h,
\end{aligned}
$$

where $\hat{\bar{P}}_{a}(k, s)$ is the double Fourier-Laplace transform of the surface pressure disturbance $P_{a}(x, t)$. The incompressible version of the Cauchy-Poisson problem (2.14)-(2.16) has already been analysed by Mei et al. (2005). Indeed, the effect of water compressibility is responsible for increased complexity and richer dynamics than in the incompressible case, as shown in the following. The solution of the boundary-value problem (2.14)-(2.16) is

$$
\hat{\bar{\phi}}(z ; k, s)=-\frac{\hat{\bar{P}}_{a}(k, s)}{\rho_{0}} \frac{\cosh \beta(z+h)}{\cosh \beta h} \frac{s}{s^{2}+g \beta \tanh \beta h},
$$

where

$$
\beta=\beta(k, s)=\sqrt{k^{2}+\frac{s^{2}}{c^{2}}}, \quad k \in \mathbb{R}, s \in \mathbb{C} .
$$

The latter expression requires choice of an appropriate Riemann surface to avoid multivaluedness, which will be discussed shortly. For now, it suffices to note that (2.17) is even in $\beta$. Then $\hat{\bar{\phi}}$ is unaffected by changes in the sign of $\beta$. The double-inverse transform of (2.17) according to (2.13) and (2.12) yields the convolution integral

$$
\phi(x, z, t)=\int_{0}^{t} \int_{-\infty}^{\infty} P_{a}(\xi, \tau) G(x-\xi, z, t-\tau) \mathrm{d} \xi \mathrm{d} \tau
$$


where

$$
\begin{array}{r}
G(x, z, t)=-\frac{1}{4 \pi^{2} \mathrm{i} \rho_{0}} \int_{-\infty}^{+\infty} e^{\mathrm{i} k x} \int_{\Gamma} \frac{\cosh (\beta(k, s)(z+h))}{\cosh (\beta(k, s) h)} \\
\times \frac{s e^{s t}}{s^{2}+g \beta(k, s) \tanh (\beta(k, s) h)} \mathrm{d} s \mathrm{~d} k
\end{array}
$$

is the Green function of the velocity potential, solution of the problem with $P_{a}(x, t)=$ $\delta(x) \delta\left(t-0^{+}\right)$. We shall now solve the inner integral of the Green function (2.20) by means of complex analysis. The poles of the integrand in the complex $s$ plane are the zeroes of

$$
s^{2}+g \beta(k, s) \tanh (\beta(k, s) h), \quad s \in \mathbb{C}, k \in \mathbb{R}
$$

Such equation has imaginary zeroes at $s=i \omega$, where $\omega \in \mathbb{R}$ satisfies the acoustic-gravity dispersion relation

$$
\omega^{2}=g \beta(k, \mathrm{i} \omega) \tanh (\beta(k, \mathrm{i} \omega) h),
$$

$\beta$ being given by (2.18). As already discussed by Stoneley (1926) and Longuet-Higgins (1950), expression (2.21) admits the solutions

$$
\beta=\beta_{0}: \omega_{0}^{2}=g \beta_{0} \tanh \beta_{0} h, \beta_{0}(k)=\sqrt{k^{2}-\frac{\omega_{0}^{2}}{c^{2}}},\left|\omega_{0}\right|<|k| c
$$

and

$$
\beta= \pm \mathrm{i} \beta_{n}: \omega_{n}^{2}=-g \beta_{n} \tan \beta_{n} h, \beta_{n}(k)=\sqrt{\frac{\omega_{n}^{2}}{c^{2}}-k^{2}},\left|\omega_{n}\right|>|k| c,
$$

where $n=1,2, \ldots$ Hence $s= \pm i \omega_{0}(k)$ and $s= \pm i \omega_{n}(k)$ are the poles of the integrand in (2.20). Note that in the incompressible limit $(c \rightarrow \infty)$ only (2.22) survives. Therefore (2.22) relates to the gravity wave, while (2.23) relates to the HA modes arising because of the compressibility of the fluid. In order to solve $(2.22)-(2.23)$, the anticipated multivaluedness of $\beta$ in (2.18) must now be resolved. The complex function $\beta$ has two imaginary branch points at $s= \pm i k c$. To make $\beta$ single valued, we shall introduce the branch cuts depicted in figure $1(a)$ and choose the Riemann sheet for which $\theta_{1} \in(-3 / 2 \pi, 1 / 2 \pi]$ and $\theta_{2} \in[-1 / 2 \pi, 3 / 2 \pi)$. With such choice, it is immediate to verify that $\beta_{0}$ in $(2.22)$ and the $\beta_{n}$ in (2.23) are positive real functions. Figure 2(a) shows the graphical solution of $(2.22)$, while figure $2(b)$ that of $(2.23)$. We are now ready to solve the inner integral of (2.20) in the complex plane. Note that no branch-cuts are required for this task since the integrand is single valued. For $t<0$, integration along the dashed circuit of figure $1(b)$, application of the residue theorem and of the Jordan lemma yields $G(x, z, t<0)=0$, which satisfies the initial rest requirement. For $t>0$, integration along the closed circuit of figure $1(b)$ and application of the same fundamental theorems of complex analysis gives $G(x, z, t)=G_{g}(x, z, t)+G_{c}(x, z, t)$, where

$$
G_{g}=-\frac{1}{\pi \rho_{0}} \int_{0}^{+\infty} \frac{\cosh \left(\beta_{0}(k)(z+h)\right)}{\cosh \left(\beta_{0}(k) h\right)} \frac{\cos k x \cos \left(\omega_{0}(k) t\right)}{1+\frac{\omega_{0}^{2}(k)}{2 c^{2} \beta_{0}^{2}(k)}\left(1+\frac{2 \beta_{0}(k) h}{\sinh \left(2 \beta_{0}(k) h\right)}\right)} \mathrm{d} k
$$

and

$$
G_{c}=-\frac{1}{\pi \rho_{0}} \sum_{n=1}^{+\infty} \int_{0}^{+\infty} \frac{\cos \left(\beta_{n}(k)(z+h)\right)}{\cos \left(\beta_{n}(k) h\right)} \frac{\cos k x \cos \left(\omega_{n}(k) t\right)}{1-\frac{\omega_{n}^{2}(k)}{2 c^{2} \beta_{n}^{2}(k)}\left(1+\frac{2 \beta_{n}(k) h}{\sin \left(2 \beta_{n}(k) h\right)}\right)} \mathrm{d} k
$$

The wave field is twofold: $G_{g}(2.24)$ represents a transient propagating gravity wave, while $G_{c}(2.25)$ describes the HA component $G_{c}=\sum_{n=1}^{\infty} G_{c n}$ made up by the HA modes 
(a) (b)
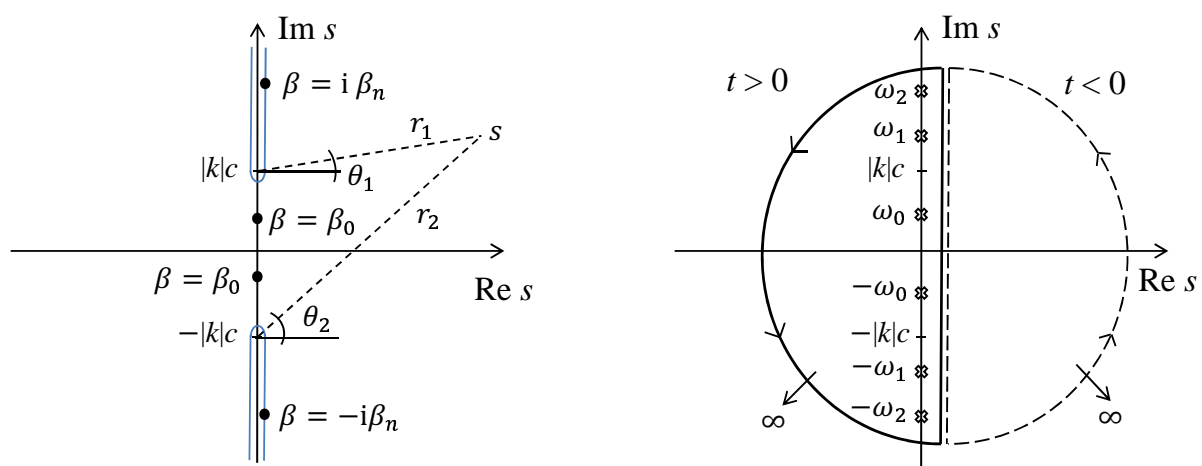

FIgURE 1. Analysis in the complex $s$ plane. (a) Branch points and branch cuts for $\beta(k, s)(2.18)$. The Riemann sheet $\theta_{1} \in(-3 / 2 \pi, 1 / 2 \pi], \theta_{2} \in[-1 / 2 \pi, 3 / 2 \pi)$ is chosen to avoid multi-valuedness. (b) Integration paths to evaluate (2.20). The bold path is for $t>0$, the dashed one for $t<0$. Recall that no branch cuts are required in $(b)$ since the integrand of $(2.20)$ is a single-valued function of $s$.
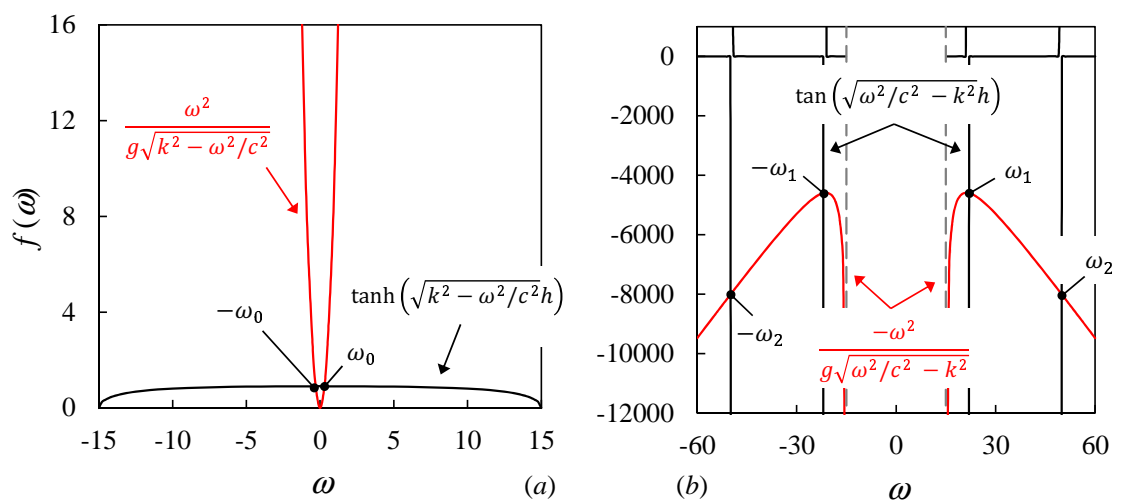

FIGURE 2. Acoustic-gravity dispersion relation in the complex $s$ plane. Graphic solutions to $(a)$ : expression (2.22); (b): expression (2.23). Parameters are: $c=1500 \mathrm{~m} / \mathrm{s}, k=0.01 \mathrm{~m}^{-1}, h=150 \mathrm{~m}$.

$G_{c n}$. Expression (2.25) is in general different from zero and shows that HA modes can be generated by the application of a surface pressure disturbance. This extends the results obtained by earlier theories as anticipated in $\S 1$. Note that $(2.25)$ is formally similar to other compressible-flow potentials, like e.g. the potential of the air flow inside a tube, open at one end and fitted with a movable piston at the other (Stoneley 1926). The solution $\phi(x, z, t)$ for a generic surface pressure distribution $P_{a}(x, t)$ can now be obtained by substituting $G=G_{g}+G_{c}$ back into the convolution integral (2.19) and solving once $P_{a}(x, t)$ is assigned. Moving forward from these foundations, in the next section we shall show with an example that HA waves can be identified as the "acoustic signature" of surface gravity waves generated by surface pressure disturbances localised in space and time. 


\section{Discussion}

Pressure fluctuations on the ocean surface are notoriously difficult to represent mathematically (Guo 1987). Exponential distributions are widely used simplifications which allow one to obtain the fundamental physical picture of the problem (Guo 1987; Mei et al. 2005). As an example, here we shall consider the acoustic-gravity wave field generated by a double Gaussian surface pressure distribution in space and time,

$$
P_{a}(x, t)=\frac{2 I_{0}}{\pi \epsilon \sigma} \mathrm{e}^{-(x / \sigma)^{2}} \mathrm{e}^{-(t / \epsilon)^{2}}, \quad x \in \mathbb{R}, t \geqslant 0,
$$

where $\epsilon$ and $2 \sigma$ are, respectively, the characteristic duration and length of the perturbation and $I_{0}=\int_{-\infty}^{\infty} \int_{0}^{\infty} P_{a}(x, t) \mathrm{d} x \mathrm{~d} t$ is the total impulse per unit $y$ width. Substituting (3.1) and the Green function components (2.24)-(2.25) into the convolution expression (2.19), after some lengthy algebra we obtain $\phi=\phi_{g}+\phi_{c}$, where

$$
\begin{aligned}
\phi_{g}(x, z, t)= & -\frac{I_{0}}{2 \pi \rho_{0}} \int_{0}^{\infty} \frac{\cosh \left(\beta_{0}(k)(z+h)\right)}{\cosh \left(\beta_{0}(k) h\right)} \frac{\mathrm{e}^{-(\sigma k / 2)^{2}}}{1+\frac{\omega_{0}^{2}(k)}{2 c^{2} \beta_{0}^{2}(k)}\left(1+\frac{2 \beta_{0}(k) h}{\sinh \left(2 \beta_{0}(k) h\right)}\right)} \\
& \times\left[a_{0}(k, t) \cos \left(\omega_{0}(k) t\right)+b_{0}(k, t) \sin \left(\omega_{0}(k) t\right)\right] \cos k x \mathrm{~d} k
\end{aligned}
$$

is the gravity wave field and

$$
\begin{aligned}
\phi_{c}(x, z, t)= & -\frac{I_{0}}{2 \pi \rho_{0}} \sum_{n=1}^{\infty} \int_{0}^{\infty} \frac{\cos \left(\beta_{n}(k)(z+h)\right)}{\cos \left(\beta_{n}(k) h\right)} \frac{\mathrm{e}^{-(\sigma k / 2)^{2}}}{1-\frac{\omega_{n}^{2}(k)}{2 c^{2} \beta_{n}^{2}(k)}\left(1+\frac{2 \beta_{n}(k) h}{\sin \left(2 \beta_{n}(k) h\right)}\right)} \\
& \times\left[a_{n}(k, t) \cos \left(\omega_{n}(k) t\right)+b_{n}(k, t) \sin \left(\omega_{n}(k) t\right)\right] \cos k x \mathrm{~d} k
\end{aligned}
$$

is the HA wave field. The $\omega_{n}(k), n=0,1, \ldots$ are again the solutions of the acousticgravity dispersion relation $(2.21)$. In (3.2) and (3.3)

$$
a_{n}(k, t)=\mathrm{e}^{-\left(\epsilon \omega_{n}(k) / 2\right)^{2}} \operatorname{erf}\left(\frac{t}{\epsilon}+\frac{\mathrm{i} \epsilon \omega_{n}(k)}{2}\right)+\text { c.c. }
$$

and

$$
b_{n}(k, t)=\mathrm{e}^{-\left(\epsilon \omega_{n}(k) / 2\right)^{2}}\left[\mathrm{i} \operatorname{erf}\left(\frac{t}{\epsilon}+\frac{\mathrm{i} \epsilon \omega_{n}(k)}{2}\right)+\operatorname{erfi}\left(\frac{\epsilon \omega_{n}(k)}{2}\right)\right]+\text { c.c. }
$$

are real functions depending on the modal order $n=0,1, \ldots$ c.c. is the complex conjugate of the preceding term. Using the expansion formula (8.254) of Gradshteyn \& Ryzhik (2007) for the error functions in (3.4) and (3.5) reveals that both $a_{n}$ and $b_{n}$ decay as $O\left(1 / \omega_{n}(k)\right)$ for $k \rightarrow \infty$. This is shown for example in figure 3(a). Hence the integrals of (3.2) and (3.3) are convergent. Note that at large time $t \gg \epsilon$, the time-dependent error functions in (3.4) and (3.5) tend to unity and both $a_{n}$ and $b_{n}$ approach values which do not depend on time. Hence $\phi_{g}(3.2)$ and $\phi_{c}(3.3)$, respectively, represent transient gravity and HA waves generated by the sudden application of the surface pressure disturbance $P_{a}$. Expressions (3.2) and (3.3) are rather complicated to study either analytically or numerically. Motivated by this fact, in the following section we shall derive simplified expressions upon solid physical grounds, which will prove very useful to obtain a clearer physical picture of the phenomenon.

\subsection{Approximated expressions}

Let us start from the hydro-acoustic potential $\phi_{c}(3.3)$. First consider the relevant dispersion relation $(2.23)$ and rewrite it as

$$
\delta_{n}(k)=\frac{g h}{\omega_{n}^{2} h^{2}}=\frac{-1}{\beta_{n} h \tan \beta_{n} h} .
$$


Supported by the fact that HA waves travel much faster than surface gravity waves (Lighthill 1978), we seek an approximation of (3.6) by assuming that gravity has little effect on the HA wave propagation. Mathematically, this translates into the assumption $\delta_{n} \ll 1$ for all $n$, whose accuracy we shall discuss shortly. An approximated form of (3.6) can now be obtained in the limit $\delta_{n} \rightarrow 0^{+}$, which then yields $\beta_{n} h \rightarrow \mathcal{B}_{n}=(2 n-1) \pi / 2$. Taylor-expanding (3.6) in the neighbourhood of $\beta_{n} h=\mathcal{B}_{n}$, using (2.23) and neglecting terms of order $O\left(\delta_{n}\right)$ and higher finally gives

$$
\omega_{n}(k) \sim \frac{c}{h} \sqrt{\mathcal{B}_{n}^{2}+(k h)^{2}},
$$

which is an approximated form of the dispersion relation for the $n$th HA mode. In order to check the degree of accuracy of such an approximation, let us substitute (3.7) back into (3.6), so that

$$
\delta_{n}(k) \sim \frac{g h}{c^{2}} \frac{1}{\mathcal{B}_{n}^{2}+(k h)^{2}} \leqslant \frac{8}{\pi^{2}} \gamma h,
$$

where $\gamma=g /\left(2 c^{2}\right) \simeq 2.18 \times 10^{-6} \mathrm{~m}^{-1}$ is the small Longuet-Higgins parameter (LonguetHiggins 1950). Expression (3.7) yields an upper bound

$$
\tilde{\delta}=\frac{4}{\pi^{2}} \frac{g h}{c^{2}}=\frac{8}{\pi^{2}} \gamma h
$$

for the sought order of approximation of (3.7). Note that $\tilde{\delta}$ is proportional to the square of the ratio between the speed of propagation $c_{g}=\sqrt{g h}$ of the leading gravity wave (Mei et al. 2005) and the speed of sound in water, which is usually much larger than $c_{g}$. For example, considering $h=150 \mathrm{~m}$ and $c=1500 \mathrm{~m} / \mathrm{s}$ gives $\tilde{\delta} \simeq 2.65 \times 10^{-4} \ll 1$. Note also that $\delta_{n} \sim n^{-2}$ at large $n$, so that the HA modes have increasingly less influence on the wave field as their modal number increases. Within this framework, Taylor-expanding (3.3) in series of $\delta_{n} \ll 1$ and neglecting the higher-order terms yields finally

$$
\begin{aligned}
\phi_{c}(x, z, t)= & -\frac{I_{0} c^{2}}{\pi \rho_{0} h^{2}} \sum_{n=1}^{+\infty}(-1)^{n} \mathcal{B}_{n} \cos \left(\mathcal{B}_{n}(1+z / h)\right) \int_{0}^{+\infty} \frac{\mathrm{e}^{-(\sigma k / 2)^{2}}}{\omega_{n}^{2}(k)} \\
& \times\left[a_{n}(k, t) \cos \left(\omega_{n}(k) t\right)+b_{n}(k, t) \sin \left(\omega_{n}(k) t\right)\right] \cos k x \mathrm{~d} k,
\end{aligned}
$$

where the $\omega_{n}$ are given by (3.7). Note that $\cos \mathcal{B}_{n}=0$ for all $n=1,2, \ldots$ Within the approximation $\tilde{\delta} \ll 1$, expression (3.9) represents a compression wave which propagates underwater, with no effect on the horizontal velocity and on the pressure at $z=0$. Let us now consider the gravity wave potential $\phi_{g}(3.2)$. Again, the large difference $c_{g} \ll c$ makes in turn the gravity wave almost independent of the HA modes, the latter travelling well ahead of the former. Within this framework, (2.22) gives $\beta_{0} \sim k$ and (3.2) simplifies to

$$
\begin{aligned}
\phi_{g}(x, z, t)= & -\frac{I_{0}}{2 \pi \rho_{0}} \int_{0}^{+\infty} \frac{\cosh k(z+h)}{\cosh k h} \mathrm{e}^{-(\sigma k / 2)^{2}} \\
& \times\left[a_{0}(k, t) \cos \left(\omega_{0}(k) t\right)+b_{0}(k, t) \sin \left(\omega_{0}(k) t\right)\right] \cos k x \mathrm{~d} k,
\end{aligned}
$$

where the well-known dispersion relation $\omega_{0}^{2}=g k \tanh k h$ is obtained. Substituting the simplified expressions (3.9) and (3.10) for $\phi=\phi_{g}+\phi_{c}$ into the dynamic boundary condition (2.7) on the free surface and developing the algebra yields finally the expression for the free-surface elevation

$$
\begin{aligned}
\zeta(x, t)= & -\frac{I_{0}}{4 \pi \rho_{0} g} \int_{0}^{+\infty} \mathrm{e}^{-(\sigma k / 2)^{2}} \omega_{0}(k)\left\{a_{0}(k, t)\left[\sin \left(k x+\omega_{0}(k) t\right)-\sin \left(k x-\omega_{0}(k) t\right)\right]\right. \\
& \left.-b_{0}(k, t)\left[\cos \left(k x+\omega_{0}(k) t\right)+\cos \left(k x-\omega_{0}(k) t\right)\right]\right\} \mathrm{d} k .
\end{aligned}
$$


The latter is an even function of $x \in \mathbb{R}$ and physically represents the sum of left- and right-going waves. The mathematical structure of (3.11) is similar to that of other transient water wave solutions (see e.g. Mei et al. 2005; Sammarco \& Renzi 2008; Renzi \& Sammarco 2012). Note that (3.5) gives $b_{0}(k, 0)=0$; hence $\zeta(x, 0)=0$ as requested. Expression (3.11) confirms that, within the approximation $\tilde{\delta} \ll 1$, the free-surface elevation is not affected by the HA modes. In other words, the HA component of the free-surface elevation is of an order $O(\tilde{\delta})$ with respect to the gravity component and hence can be neglected to calculate $\zeta$. This result would not hold if the forcing surface pressure $P_{a}$ was a travelling wave whose dispersion relation matched that of any HA mode. In that circumstance, resonant effects would occur and the surface displacement would be dominated by the HA modes. Furthermore, note that the HA component (3.9) can have effects on the vertical velocity which are relevant to the generation of microbaroms in the atmosphere (Waxler \& Gilbert 2006; Ardhuin \& Herbers 2013).

Employing a similar procedure to that used for $\zeta$, but with the Bernoulli equation (2.4), yields the dynamic pressure $p_{d}=p_{g}+p_{c}$, where

$$
\begin{aligned}
p_{g}(x, z, t)= & -\frac{I_{0}}{4 \pi} \int_{0}^{+\infty} \frac{\cosh k(z+h)}{\cosh k h} \mathrm{e}^{-(\sigma k / 2)^{2}} \omega_{0}(k)\left\{a _ { 0 } ( k , t ) \left[\sin \left(k x+\omega_{0}(k) t\right)\right.\right. \\
& \left.\left.-\sin \left(k x-\omega_{0}(k) t\right)\right]-b_{0}(k, t)\left[\cos \left(k x+\omega_{0}(k) t\right)+\cos \left(k x-\omega_{0}(k) t\right)\right]\right\} \mathrm{d} k+ \\
& +\frac{2 I_{0}}{\pi^{3 / 2} \epsilon} \mathrm{e}^{-(t / \epsilon)^{2}} \int_{0}^{\infty} \frac{\cosh k(z+h)}{\cosh k h} \mathrm{e}^{-(\sigma k / 2)^{2}} \cos k x \mathrm{~d} k
\end{aligned}
$$

is the gravity pressure component and

$$
\begin{aligned}
p_{c}(x, z, t)= & -\frac{I_{0} c^{2}}{2 \pi h^{2}} \sum_{n=1}^{+\infty}(-1)^{n} \mathcal{B}_{n} \cos \left[\mathcal{B}_{n}(1+z / h)\right]\left\{\int _ { 0 } ^ { + \infty } \frac { \mathrm { e } ^ { - ( \sigma k / 2 ) ^ { 2 } } } { \omega _ { n } ( k ) } \left\{a_{n}(k, t)\right.\right. \\
& \times\left[\sin \left(k x+\omega_{n}(k) t\right)-\sin \left(k x-\omega_{n}(k) t\right)\right]-b_{n}(k, t)\left[\cos \left(k x+\omega_{n}(k) t\right)\right. \\
& \left.\left.\left.+\cos \left(k x-\omega_{n}(k) t\right)\right]\right\} \mathrm{d} k-\frac{8}{\sqrt{\pi} \epsilon} \mathrm{e}^{-(t / \epsilon)^{2}} \int_{0}^{\infty} \frac{\mathrm{e}^{-(\sigma k / 2)^{2}}}{\omega_{n}^{2}(k)} \cos k x \mathrm{~d} k\right\}(3.13
\end{aligned}
$$

is the HA pressure component, i.e. the sought underwater compression wave. Note that the gravity component $p_{g}(3.12)$ is the sum of two integral terms. The first one is associated with the transient propagating surface wave (3.11), while the second decays exponentially with time like $P_{a}(3.1)$ and therefore is a source-related term. Also, the HA pressure $p_{c}(3.13)$ is the sum of a transient propagating underwater compression wave and an evanescent term which decays exponentially with time. The separation between propagating and evanescent acoustic waves was also obtained in previous work analysing R and AG solutions (Kibblewhite \& Ewans 1996; Ardhuin \& Herbers 2013). Note that the vertical cosine dependence of the HA pressure modes in (3.13) implies the HA pressure be largest at the bottom, despite the forcing is applied on the free surface! Incidentally, further approximated expressions of the propagating waves may be obtained by using the method of stationary phase as in Stiassnie (2010).

The present model yields new results with respect to established incompressible and compressible theories. The incompressible theory (Mei et al. 2005) naturally fails to capture the underwater HA pressure wave travelling ahead of the surface gravity wave. Previous compressible models (Longuet-Higgins 1950; Ardhuin \& Herbers 2013; Ardhuin et al. 2013), though providing a basis for the present analysis, did not consider the firstorder effects of compressibility in the co-generation of HA and gravity waves directly by a sudden, localised pressure disturbance. Our new results show that the HA pressure signal $p_{c}$ (3.13) can be interpreted as the "acoustic signature" of the surface gravity wave 

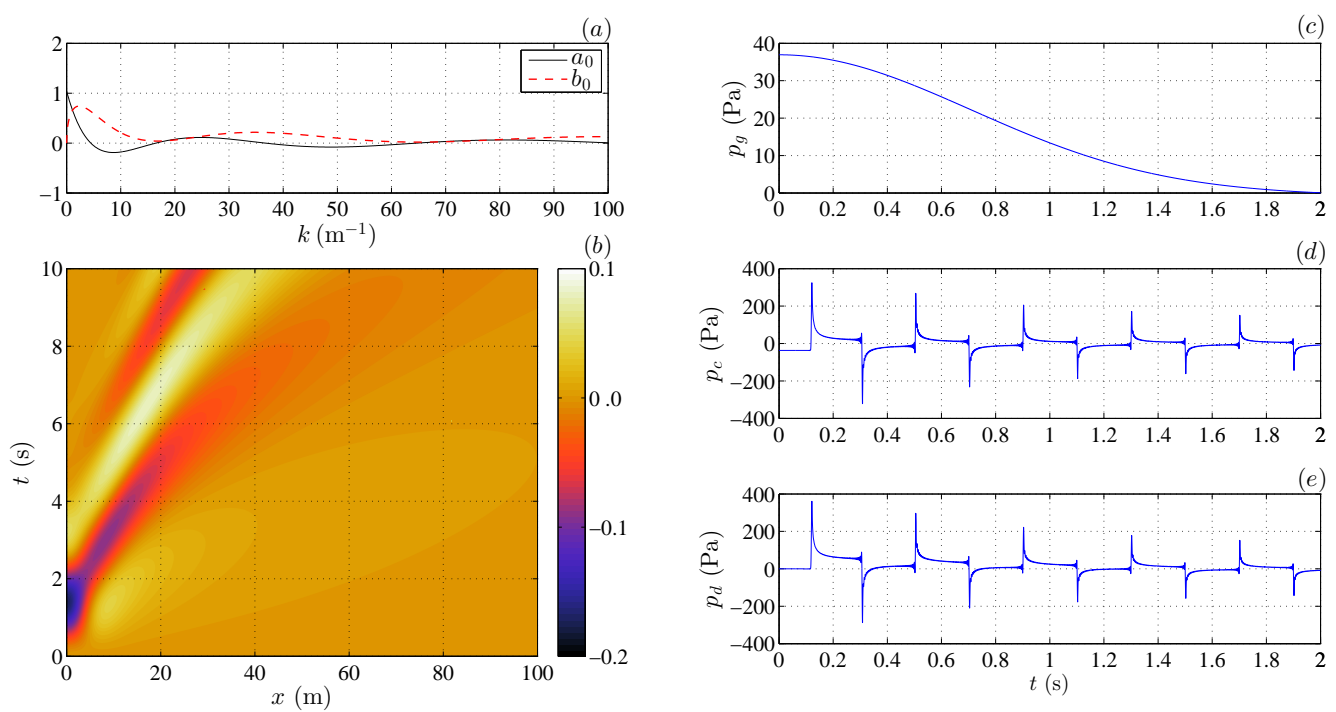

Figure 3. (a) Behaviour of $a_{0}(3.4)$ and $b_{0}(3.5)$ versus $k$ for $t=0.5 \mathrm{~s}$ and $\epsilon=1 \mathrm{~s}$. (b) Space-time density plot of the free-surface elevation $\zeta(3.11)$ generated by the double-Gaussian pressure distribution $P_{a}(3.1)$. (c) Time series of the gravity pressure component $p_{g}(3.12)$ at $\left(x_{0},-h\right)=(100,-150) \mathrm{m}$. $(d)$ Time series of the HA pressure component $p_{c}(3.13)$ at $\left(x_{0},-h\right)$. (e) Time series of the total dynamic pressure $p_{d}=p_{g}+p_{c}$ at $\left(x_{0},-h\right)$. The parameters for figures (b) $-(e)$ are: $I_{0}=1.57 \times 10^{4} \mathrm{~N} \mathrm{~s} / \mathrm{m}, \sigma=5 \mathrm{~m}, \epsilon=1 \mathrm{~m}$ and $h=150 \mathrm{~m}$. The first 50 acoustic normal modes are considered. Note that $p_{d}=0$ at $t=0$ in accordance with the condition (2.11) of hydrostatic pressure distribution in the inner layers of the fluid at $t=0$.

$\zeta$ (3.11) generated by the application of a surface pressure disturbance localised in space and time. A numerical example further illustrates this novel result.

\section{Numerical example}

Figure 3(b) shows the space-time density plot of the free-surface elevation $\zeta$ (3.11), generated by the double-Gaussian perturbation $P_{a}(3.1)$ of parameters $I_{0}=1.57 \times 10^{4} \mathrm{~N}$ $\mathrm{s} / \mathrm{m}, 2 \sigma=10 \mathrm{~m}$ and $\epsilon=1 \mathrm{~s}$, for which $P_{a}(0,0)=2000 \mathrm{~Pa}$. This value roughly corresponds to the pressure $\rho_{\text {air }} v^{2} / 2$ applied by the air moving at speed $v=55 \mathrm{~m} / \mathrm{s}$, with $\rho_{\text {air }} \simeq$ $1.2 \mathrm{~kg} / \mathrm{m}^{3}$. The water depth is $h=150 \mathrm{~m}$. Soon after the surface pressure is applied, a trough forms close to the origin. At larger time, new surface waves are generated. Leading long waves travel faster and are followed by tails of shorter waves, as predicted also by the incompressible model of Mei et al. (2005). However, the limits of the incompressible theory appear clearly in figures $3(c)$ and $3(d)$. These show, respectively, the gravity (3.12) and HA (3.13) components of the dynamic pressure $p_{d}$, evaluated at the bottom $z=-h$, at $x=x_{0}=100 \mathrm{~m}$, that is $x_{0} /(2 \sigma)=10$. Numerical analysis reveals that the gravity component $p_{g}$ is much smaller than the HA term $p_{c}$. Figure $3(e)$ shows the time series of the dynamic pressure $p_{d}=p_{g}+p_{c}$ at the bottom. The signal reaches the observation point $\left(x_{0},-h\right)$ at $t \simeq 0.12 \mathrm{~s}$, that is roughly $t \simeq \sqrt{x_{0}^{2}+h^{2}} / c$. The fluid elements are initially compressed and then relaxed by the first pressure wave. The latter is followed by a train of waves with decreasing amplitude (see again figure $3 e$ ), generated by the boundary reflections of the initial pressure perturbation. Such reflections are characterised by longer travel times than the first signal and, because of their transient nature, experience an increasingly stronger attenuation with time. The average zero-up- 
crossing frequency of the pressure wave is $f \simeq 2.5 \mathrm{~Hz}$. This corresponds to the cut-off frequency $f_{0}=c /(4 h)$ of the first HA mode, which is indeed dominant (Eyov et al. 2013). Such a frequency falls outside the typical range of ultra-low-frequency (ULF) R and AG waves produced by second-order wave-to-wave interactions described by Ardhuin et al. (2013). Therefore HA waves generated by sudden surface pressure disturbances seem to possess a peculiar frequency signature which favourably sets them apart from $\mathrm{R}$ and AG signals. Note also from figure $3(e)$ the characteristic spiky behaviour of the pressure signal, which pulsates in a saw-chain fashion. This happens since the HA pressure wave is little influenced by the restoring action of gravity. The latter smooths the free-surface elevation but not the pressure signal, which then reveals its original impulsive generation mechanism also at later instants. Finally, comparison of figures $3(b)$ and $3(e)$ shows that the first HA pressure signal reaches the observation point $\left(x_{0},-h\right)$ at $t \simeq 0.12 \mathrm{~s}$, much in advance than the arrival at $x_{0}$ of the surface gravity wave, which occurs at about $t=5 \mathrm{~s}$. Therefore, the numerical example of figure (3) confirms that HA pressure waves exist as precursors of the surface gravity waves generated by sudden surface pressure variations. For example, they remind of the capillary forerunners of the initial excitation induced in a thin layer of mercury before the arrival of the gravity perturbation (Falcon et al. 2003). However, while the latter are observed on the fluid surface, the HA waves are to be detected below the surface, e.g. by means of modern hydrophones. We also carried out calculations at larger distance from the origin. Moving away from the source, both the surface gravity wave and the HA pressure signal attenuate more strongly in our simulations than in those of Stiassnie (2010) and Sammarco et al. (2013) for earthquakegenerated waves. This is likely because large-scale earthquakes yield a stronger energy release than localised atmospheric disturbances. This fact does not hinder the practical importance of our results: HA waves could be used to design alert systems for coastal flooding generated by nearby atmospheric perturbations.

\section{Conclusions}

We showed that sudden, localised pressure changes on the free surface of an ocean can generate a system of hydro-acoustic (HA) pressure waves which are the precursors of the associated surface gravity waves. This result provides an advancement on established incompressible theories, which naturally fail to capture HA waves, and on previous compressible models, which did not consider such dynamics. Numerical calculations show that the underwater compression waves can be interpreted as the "acoustic signature" of the surface gravity wave. This dynamics can be exploited to design innovative coastal flooding warning systems based on the detection of underwater compression waves as the forerunners of the surface gravity waves. Additional work is being carried out to extend this fundamental theory to three-dimensions, uneven elastic bottom (Eyov et al. 2013) and travelling disturbances, for which a numerical approach, in the style of Sammarco et al. (2013), would be required.

The work of E.R. is funded by the AXA Research Grant "Underwater acoustic sensing for detection, early warning and increased risk awareness of storm surges with application to Ireland". F.D. is supported by the ERC-2011-AdG 290562 - MULTIWAVE. Discussions with Dr G. Bellotti, Dr C. Cecioni, Dr M. Onorato and Professor O.C. Rodriguez are kindly acknowledged. 


\section{REFERENCES}

Ardhuin, F. \& Herbers, T. H. C. 2013 Noise generation in the solid Earth, oceans and atmosphere, from nonlinear interacting surface gravity waves in finite depth. J. Fluid Mech. 716, 316-348.

Ardhuin, F., Lavanant, T., Obrebski, M., Marié, L., Royer, J.-Y., D’Eu, J.-F., Howe, B.M., Lukas, R. \& Aucan, J. 2013 A numerical model for ocean ultra low frequency noise: wave-generated acoustic-gravity and rayleigh modes. J. Acoust. Soc. Amer. 134, $3242-3259$.

Eyov, E., Klar, A., Kadri, U. \& Stiassnie, M. 2013 Progressive waves in a compressible ocean with an elastic bottom. Wave Motion 50, 929-939.

Falcon, E., Laroche, C. \& Fauve, S. 2003 Observation of Sommerfeld precursors on a fluid surface. Phys. Rev. Lett. 91 (6), 064502.

Gradshteyn, I.S. \& Ryzhik, I.M. 2007 Tables of Integrals, Series and Products. Academic Press.

GuO, Y.P. 1987 Waves induced by sources near the ocean surface. J. Fluid Mech. 181, 293-310.

Hasselmann, K. 1963 A statistical analysis of the generation of microseisms. Rev. Geophys. $1(2), 177-210$.

Kibblewhite, A.C. \& Ewans, K.C. 1996 Wave Interactions as a Seismo-Acoustic Source. Springer.

Lighthill, J. 1978 Waves in Fluids. Cambridge University Press.

Longuet-Higgins, M.S. 1950 A theory of the origin of microseisms. Phil. Trans. Roy. Soc. London A 243, 1-35.

MeI, C.C. 1997 Mathematical Analysis in Engineering. Cambridge University Press.

Mei, C.C., Stiassnie, M. \& Yue, D.K.-P. 2005 Theory and applications of ocean surface waves. World Scientific.

Renzi, E. \& SAmmarco, P. 2012 The influence of landslide shape and continental shelf on landslide generated tsunamis along a plane beach. Nat. Hazards Earth Syst. Sci. 12, 15031520 .

Sammarco, P., Cecioni, C., Bellotti, G. \& Abdolali, A. 2013 Depth-integrated equation for large-scale modelling of low-frequency hydroacoustic waves. J. Fluid Mech. 722, 1-10.

Sammarco, P. \& Renzi, E. 2008 Landslide tsunamis propagating along a plane beach. J. Fluid Mech. 598, 107-119.

Stiassnie, M. 2010 Tsunamis and acoustic-gravity waves from underwater earthquakes. J. Eng. Math. 67 (1-2), 23-32.

Stoneley, M.A. 1926 The effect of the ocean on Rayleigh waves. Mon. Not. Roy. Astron. Soc. Geophys. Suppl. 1, 349-356.

WAXler, R. \& GilBeRT, K.E. 2006 The radiation of atmospheric microbaroms by ocean waves. J. Acoust. Soc. Amer. pp. 2651-2664.

Wilson, J.D. \& MAKRIs, N.C. 2008 Quantifying hurricane destructive power, wind speed, and air-sea material exchange with natural undersea sound. Geophys. Res. Lett. 35 (10), L10603. 